\title{
Research Knowledge Translation Activities in Africa; An Outlook of the Opportunities and Obstacles
}

\section{Jack Ogony ${ }^{1 *}$, Barclay Obiero', Becky Tukhuwa ${ }^{2}$, Chakuya Gondwe ${ }^{2}$, Edith Kampeta ${ }^{3}$, Carla Orner ${ }^{4}$ and Tena Tiruneh ${ }^{4}$}

\author{
${ }^{1}$ PHIA Fellows (Kenya), ${ }^{2}$ PHIA Fellows (Malawi), ${ }^{3}$ PHIA Fellow (Rwanda), ${ }^{4}$ United States of America \\ Heart to Heart International, P.O. Box 15566. Lenexa, KS 66285
}

*Corresponding author details: Jack Ogony; Ogonyjack@gmail.com

\begin{abstract}
Health systems have failed globally to use research evidence optimally in view of ineffective knowledge translation. Knowledge translation is the effective and timely incorporation of evidence-based information into the practices of health professionals to effect optimal health outcomes. With the growing demands on health care resources and accountability, greater emphasis is being placed on generating knowledge that can have a practical impact on decision making. This review gives an outlook of the opportunities and obstacles to knowledge translation in African context. We searched for articles published in English on the World Health Organization website, peer reviewed articles on Google Scholar, PubMed and Science Direct databases search engines since 2000 in Sub-Saharan Africa A total of 3,020 papers were analyzed and the outcomes reported. The knowledge transfer literature in African countries is widely distributed, problematically diverse and growing. However, significant disparities exist between reports in different countries while there are many settings without published evidence of local knowledge translation. Our review also highlighted the lack of meaningful investment and follow up after research findings. Barriers affecting knowledge translation includes insufficient skills and capacity to conduct knowledge transfer activities, time constraints and a lack of resources. We identified substantial geographical gaps in knowledge translation and evidence that need to boost local research capacities in Africa. Evidence mapping is a useful approach that can assist local decisionmaking to enhance knowledge transfer into a policy. We recommend the inclusion of knowledge translation advisors who are the advocates of the knowledge translation during protocol development.
\end{abstract}

Keywords: evidence-based; dissemination; obstacles; opportunities; policymaking; public health

\section{INTRODUCTION}

Globally, it has been acknowledged that health research knowledge translation (KT) into policy and practice is a significant step in strengthening the performance of health systems. However, evidence indicates that whilst there are numerous promising research findings, they are rarely translated or takes a longer time to be translated into health policy to reach the final end users. According to World Health Organization (WHO), knowledge translation (KT) is defined as "the exchange, synthesis and application of knowledge by relevant stakeholders to accelerate the benefits of global and local innovations in strengthening health systems and improving people's health"(1),while at CanChild, knowledge translation was defined as the creation, synthesis, and dissemination of research evidence in a user friendly and accessible way that is relevant and meaningful for youth, families, and health care providers. This process involves two essential phases; knowledge creation and knowledge application (2). Since the emergence of the evidence-based medicine movement in the 1990s, the importance for researchbased knowledge to inform health policy and practice is still a public health problem $(3,4)$.
Annually, billions of dollars are spent in both public and private sectors on biomedical, clinical, and health services research, undergraduate healthcare professional training and continuing professional development, quality improvement, patient safety, and risk management (5). Knowledge generated through health research has the potential to improve health outcomes, promote service delivery and strengthen health systems functioning (4, 6-8). However, there is no consistent finding from the health services literature to ascertain the obstacles /hindrances to translate research findings into health policy and practice (5). Despite burgeoning interest in this know-do gap, the translation process remains slow, haphazard and unpredictable, resulting in reduced health gains vis-á-vis global investment in research (9). In low-resource, highdisease settings, such as those found in many African countries, the consequences of ineffective KT are amplified, emphasizing the need for health system decision-makers to justify their decisions based on high-quality evidence (10). The African network for Evidence-Informed Policy Networks (EVIPNet) (figure 1), a WHO program which is responsible for 
supporting evidence-informed policymaking in lowand middle-income countries consists of twelve African countries; Burkina Faso, Cameroon, Cape Verde, Central African Republic, Ethiopia, Malawi, Mozambique, Nigeria, Senegal, Uganda, Tanzania and Zambia, all consented to Research for Health:
A strategy for the African region 2016-2025 requesting member states to setup Knowledge Translation Programs to facilitate African governments support this initiatives. This is one of the key mechanisms introduced by WHO to reduce the research to policy gap (11).

\section{EVIPNet Into Practice}

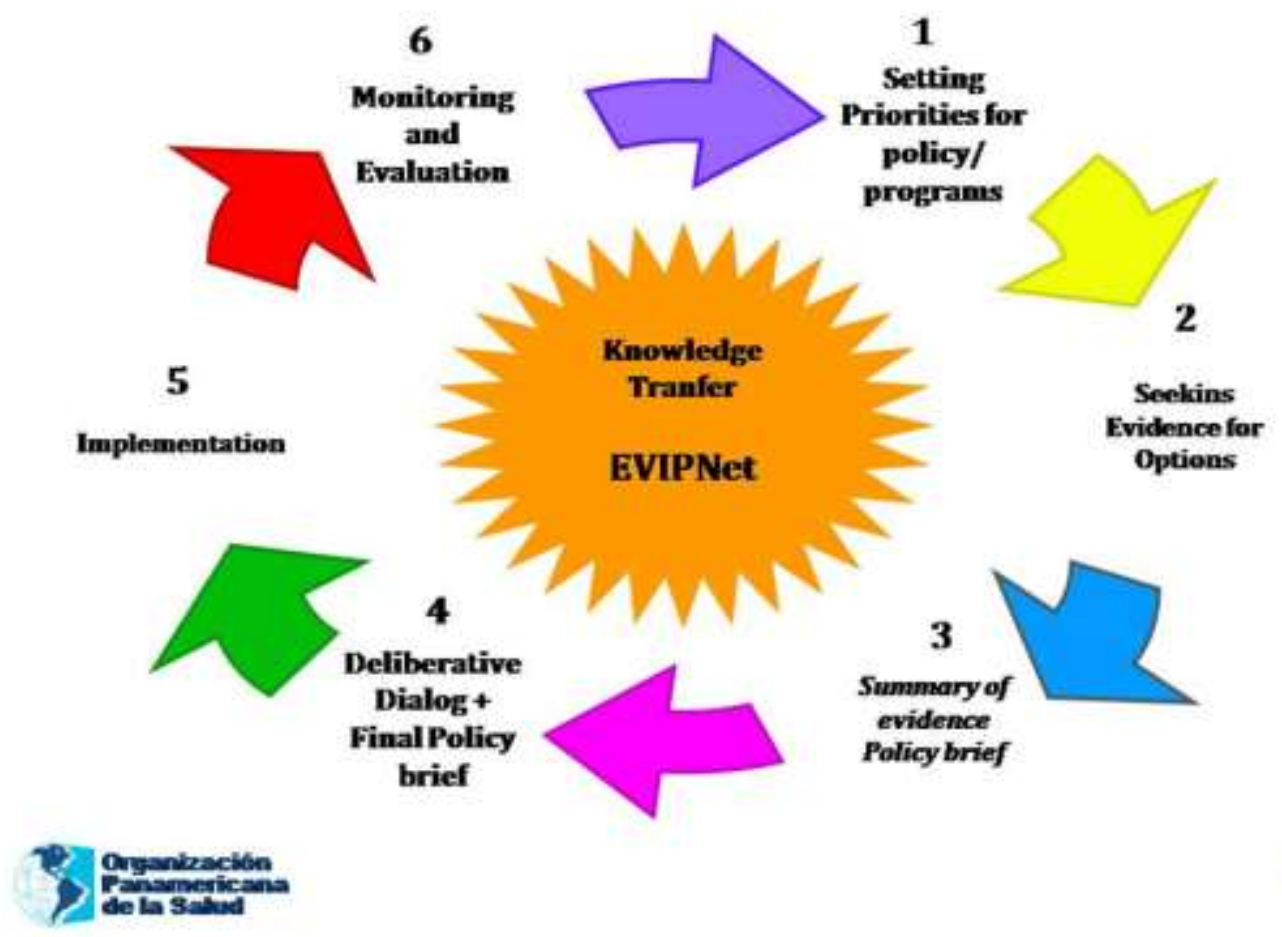

FIGURE 1 : EVIPNet in Practice

The Kenya Health and Research Observatory (KHRO), Kenya Harmonized Health Facility Assessment (KHFA) and other Ministry of Health strategic promotes evidence-informed decision by improving access and use of research and health information (12). The evidence-based on Knowledge Translation Programs (KTP) in Burundi, Cameroon, Kenya, Malawi, Nigeria, Rwanda, Tanzania, Uganda and Zambia showed that KTP requires a strong leadership. Few individuals have the essential qualities for leading a KTP as was the case for Zambia (13). However, engagement and communication activities that sensitize stakeholders about the benefits of the KTP with adequate funding have yielded better results in Malawi (Knowledge Translation Platform in Malawi (KTPMalawi)) whose funding was not always consistent but, after seeing the impact and results of KTP, the Ministry of Health (MoH) in Malawi helped fund the program. Through ongoing leadership, as well as financial and administrative support, the Malawi Ministry of Health has strongly signaled its intention to utilize a knowledge translation platform methodology to support evidence-informed national health policy (14)

With the perpetual emergence of infectious diseases which threaten human health and public safety such as the current novel SARS-CoV-2 and COVID-19 (15), there is need for urgency in steady translating research findings into a palatable policy by end users.
However, despite an increasing mobilization of researchers and donor-funding agencies, there is a persistent gap between available scientific knowledge and its use $(16,17)$. The scientific literature on KT still provides little evidence on the processes that lead to the use of scientific knowledge in a given context and, on its effectiveness, $(17,18)$. In fact, KT strategies present the characteristics of complex social interventions: they are based on several theories such as organizational change, collaboration, individual behaviour among others and they imply the commitment and participation of these actors and their organizations (19). Thus, more studies are needed to understand how research could play a role in policy and practice, especially in Africa, where a lot of research are implemented but still few initiatives to support evidence informed policy and practice are implemented and evaluated. To date, KT has focused primarily on information exchange between researchers and policy makers as opposed to study participants or the clients; however, there are principles that may be relevant in this new context. This gap in literature becomes even more salient in the context of public health research where research can reveal particular misunderstandings or shortcomings in knowledge that threatens to severely compromise participants' health (20) 
Research organizations and research funders can probably be excused for not basing their activities on research evidence until now, however, because the variety of relevant questions, target audiences, and disciplinary perspectives and methodological approaches used in empirical studies has made the identification of take-home messages from this field of research an exceedingly difficult task (7). The framework provides an overall approach to knowledge transfer that can be evaluated over long periods of time, as well as specific elements that can be evaluated and fine-tuned over shorter periods of time. KT describes how knowledge and ideas move from the knowledge source to the potential users of that knowledge (2). The Research Councils encourage knowledge transfer by supporting schemes and activities to transfer good ideas, research results and skills. One important problem is how to make better use of publicly funded research and development (10).

Knowledge Translation has four significant elements according to Canadian Institute of Health Research (CIHR) (21);

\section{(1) Synthesis}

Synthesis, in this context, means the contextualization and integration of research findings of individual research studies within the larger body of knowledge on the topic. A synthesis must be reproducible and transparent in its methods, using quantitative and/or qualitative methods. It could take the form of a systematic review, follow the methods developed by the Cochrane Collaboration, result from a consensus conference or expert panel or synthesize qualitative or quantitative results. Realist syntheses, narrative syntheses, meta-analyses, meta-syntheses and practice guidelines are all forms of synthesis.

\section{(2) Dissemination}

Dissemination involves identifying the appropriate audience and tailoring the message and medium to the audience. Dissemination activities can include such things as summaries for / briefings to stakeholders, educational sessions with patients, practitioners and/or policy makers, engaging knowledge users in developing and executing dissemination/implementation plan, tools creation, and media engagement.

\section{(3) Exchange}

The exchange of knowledge refers to the interaction between the knowledge user and the researcher, resulting in mutual learning. The Canadian Foundation for Healthcare Improvement (CFHI) define knowledge exchange as "the collaborative problem-solving between researchers and decisionmakers that happens through linkage and exchange. Effective knowledge exchange involves interaction between decision-makers and researchers and results in mutual learning through the process of planning, producing, disseminating, and applying existing or new research in decision-making."
(4) Ethically-sound application of knowledge

Ethically-sound KT activities for improved health are those that are consistent with ethical principles and norms, social values, as well as legal and other regulatory frameworks - while keeping in mind that principles, values and laws can compete among and between each other at any given point in time. The term application is used to refer to the iterative process by which knowledge is put into practice

The "exchange model" of KT requires that there should be some kind of relationship between researcher and the policy makers (22). Such relationships are characterized by regular exchanges of information, ideas and experience. More research is needed to fully explore the benefits of such relationship-building. However, some evidence is emerging that when researchers have an ongoing relationship with public policymakers, members of this particular audience are more likely to use research knowledge in their decision-making (23). Relationship building is best undertaken when research of interest to the audience is ongoing, and more findings are expected and can be transferred over time. The researcher may produce new information that helps the user make better informed decisions; the audience may in turn inform the researchers things they want to know - for example, what happened when research knowledge was used in the "real world" and what research priorities should be targeted. Building and sustaining relationships with ever-changing audiences requires a long-term investment of resources. Although lack of effectiveness or lack of success of knowledge translation in various regions, countries, industries, or forms of cooperation exist, the question of translation barriers, their impact in the process so far has not been of central interest in economic research.

\section{METHODOLOGY}

We conducted a narrative literature review on significance of research knowledge transfer to health policy- makers in Africa. The review also searched on the possible obstacles hindering the health policymakers in implementing the research findings across the Sub-Saharan Africa (SSA) countries. During the period 2020-2021, we searched for articles published in English on the WHO website, peer reviewed articles on Google Scholar and PubMed and Science Direct databases search engines written within SSA. We used keywords that included: 'research knowledge translation'; 'obstacles to KT'; 'policy makers'; 'significance of KT'. All the authors reviewed the literature from the bibliography and drafted the first draft of the paper. A total of 3,020 policy papers were analyzed and the outcomes reported. Some of the articles listed on our reference list are those quoted directly but do not necessarily represent the sources reviewed. Consistent with narrative review methodology, some steps such as quality appraisal of the evidence that would occur with a systematic review were omitted. 
TABLE 1: Eligibility criteria

\begin{tabular}{|l|l|}
\hline \multicolumn{1}{|c|}{ Eligibility criteria } & \multicolumn{1}{c|}{ Exclusion criteria } \\
\hline $\begin{array}{l}\text { Reviews on importance and obstacles on } \\
\text { knowledge transfer. }\end{array}$ & $\begin{array}{l}\text { Non-systematic literature reviews or discussion } \\
\text { papers. }\end{array}$ \\
\hline Reviews that were written in the English. & $\begin{array}{l}\text { Other types of articles such as protocols, editorial } \\
\text { comments, conference abstracts, and policies. }\end{array}$ \\
\hline Between the period of the year 2000-2021 & $\begin{array}{l}\text { Publications where full-text versions could not be } \\
\text { obtained. }\end{array}$ \\
\hline
\end{tabular}

\section{DISCUSSION \\ Knowledge translation matters!}

Graham and colleagues developed conceptual framework to ease the process of knowledge transfer and application of research findings (2). This model has been adopted by the Canadian Institutes of Health Research (CIHR) as the accepted model for promoting the application of research and for the process of knowledge translation. In this model, the process of translating knowledge to action is an iterative, dynamic and complex process. It concerns the creation and action cycle of knowledge. Although it is drawn as a cycle, users may need to use the phases out of sequence, depending on the project. This approach demands that it is essential that the end-users of the knowledge are included to ensure that the knowledge and its subsequent implementation are relevant to their needs, this has not been fully embraced by the African set up. According to this framework, Knowledge creation, or the production of knowledge, is composed of; knowledge inquiry, synthesis of knowledge and creation of knowledge tools. As knowledge is distilled through each stage in the knowledge creation process, the resulting knowledge becomes more synthesized and potentially more useful to end-users (24).

With an aim to effectively translate research results from the bench to patient's bedside, in 2016, Elizabeth, Richelle, Furqan and Helen launched the Canadian Retinoblastoma patient engagement strategy. The strategy is governed by a board made up of patients, health care professionals, and researchers. The goal of the board is to improve the quality of research related to retinoblastoma (25). The Canadian Research Realm for Rare Eye Disease (CRRAB) was established with the goal of recruiting new members and improving the quality of life for people with retinoblastoma in Canada. Like other advocates, members of the CRRAB were promoted through social media. The organization's first annual meeting was attended by interested members. The meeting provided members with an opportunity to develop their own patient-engagement activities. Since its inception, the CRRAMB has developed a research registry that is a sustainable vehicle to engage diverse groups of patients in research. This registry is also used to set the top 10 research priorities for Canada (26).
During the 2019 Health Technology Assessment (HTA) Link Conference, a pre-conference session was held to discuss the various challenges faced by the professions in Asia and selected African countries including Kenya and Zambia. The concept of the HTA has been acknowledged as a tool for setting up an effective Universal Health Coverage system. The discussions were focused on three main areas: 1) the dissemination of knowledge about the various phases of the HTA process, 2) the establishment of effective and efficient HTA agencies, and 3) the dissemination of knowledge about the various aspects of the HTA profession. The objective of this engagement was to identify and build capacity for individuals and institutions to translate research into action in Bhutan, Zambia and Kenya. Establishing a legal mandate for HTA is an important step in addressing the various challenges that arise in building local technical capacity. Establishing strategic communication channels and partnerships with various stakeholders can help address these issues (17).

Some experiments were implemented in different countries in West Africa by implementing the unique initiative called Knowledge Management and Transfer Unit (KMTU). For example, a rapid response team was created by the African Population and Health Research Center (APHR) in seven countries of West-Africa: Kenya, Rwanda, Zambia, Malawi, Sierra Leone, Burkina Faso and Liberia (27). The mandate of these teams was to facilitate the provision, in a timely manner, of rapidly produced, high-quality, synthesized evidence. Other initiatives of this type were integrated in a platform aimed to provide quick access for policymakers in Burkina Faso to high quality research evidence about health systems (28). But unfortunately, none of these initiatives were directly attached to the Minister's cabinet with a clear mandate to inform all public decisions on health.

The synthesis approach published in 2016 by the Alliance for Useful Evidence (AUE) (Table 2) focused on the effectiveness of six different mechanisms aimed at increasing the use of research by decisionmakers which presented the results of 23 systematic reviews in the field of $\mathrm{KT}$, supplemented by a scoping review in the broader social science literature still suffices since no other result has ever contradicted Langer's conclusions (18). 
TABLE 2 : different mechanisms aimed at increasing the use of research

\begin{tabular}{|c|l|}
\hline \multicolumn{2}{|c|}{ Six mechanisms aimed at increasing the use of research } \\
\hline 1. & $\begin{array}{l}\text { Interactions between researchers and decision -makers must be considered a priority } \\
\text { awareness and build agreement on research priorities. }\end{array}$ \\
\hline 2. & $\begin{array}{l}\text { Use strategies like social marketing and awareness -raising campaigns to develop more } \\
\text { attitudes towards research within the ministry }\end{array}$ \\
\hline 3. & $\begin{array}{l}\text { Research a consensus on the issues of interest to the ministry by using participatory and } \\
\text { collaborative process and intervention proven effective. }\end{array}$ \\
\hline 4. & $\begin{array}{l}\text { Improve access and communicate results in an appropriate format, in a timely manner and } \\
\text { using a variety of means adapted to users' context. }\end{array}$ \\
\hline 5. & $\begin{array}{l}\text { Train researchers, to be able to communicate clearly to a non-research audience and users to } \\
\text { be able to access and understand research results. }\end{array}$ \\
\hline 6. & $\begin{array}{l}\text { Change structures that influence decision-making process to promote evidence use by } \\
\text { policymakers. }\end{array}$ \\
\hline
\end{tabular}

\section{Obstacles to knowledge translation}

The interactive-recursive model of knowledge transfer can serve as a basis for the identification and analysis of potential transfer barriers. Based on the assumptions that first; research projects lead to positive results respectively solutions are found for previously defined problems, and secondly; the existence of these solutions is known to a circle of potential knowledge-takers, barriers of knowledge transfer (24). Study done by Dagenais, C. (2021) in Burkina Faso to determine research use at the Ministry of Health $(\mathrm{MoH})$ revealed that apart from the sometimes unwillingness to support research, the main obstacles identified were; the difficulties of accessing research results, the insufficiency in dissemination and lack of action plans to apply those results were rarely proposed in the findings (22); Given that "we can't use what we don't know exists," the issue of access to research results is fundamental, and such access must be timely. However, the majority of respondents reported having difficulty identifying studies that could help them make decisions. This was, according to some, partly due to the fact that researchers often are not concerned about communicating their results in any way other than through scientific publications: "...the researcher does this to earn his stripes; they'll disseminate [results] elsewhere, but not here...." Even though respondents knew a significant number of studies were being conducted, there was no registry they could consult to identify those that might be useful to them in their circumstances. They highlighted the absence of any clear communication strategy for research.

To explore the impact of knowledge translation platforms (KTPs) in achieving health system development goals in Uganda and Cameroon, a comparative case study showed that KTPs could influence the way health policy is formulated. It could also improve access to policy-relevant evidence by introducing safe-harbour discussions. The case study shows how two KTPs influenced different policy decisions through their interactions with the public (20).
The other obstacle is related to the poor researchers 'conclusions and the fact that they often do not make recommendations regarding how to apply the findings, this reduces the likelihood that the studies conducted will lead to changes but rather will retain status quo.

Since "in [training] schools there is no attention given to use", support is often needed to implement changes that could result from a study.

Dagenais, C. (2021) indicated that use of poor modes of communication such as specialized jargon could be difficult to grasp. Likewise, initiating of studies that were not relevant to the health problems or relevance of available studies that are not always in line with the realities confronting the Ministry: "...research is conducted that is useful to the researcher, but not necessarily to the department." They also highlighted the difficulties of funding not only the research itself, but also its implementation.

According to John Lavis, there are five key principles that have provided a very accessible formulation of the evidence on knowledge transfer which he expresses as five principles that should guide knowledge transfer to maximize uptake. These principles can be expressed as five basic questions: What (is the message)? To whom (audience)? By whom (messenger)? How (transfer method)? With what expected impact (evaluation)? (19)

The Ottawa Model of Research Use (OMRU) provides a useful framework that gives direction for tailoring interventions. The principle of OMRU is that success rests with tailoring KT strategies to the salient barriers and supports found within the setting. According to the model, barriers and supports found in the practice environment and the evidence-based innovation be assessed and then the KT strategy tailored and executed (29)

Another glaring obstacle that KT faces in Africa is the illogical setting of research priorities; Different indicators of impact are used for practitioners and policy makers than for scientists. 
Researchers, especially those in academic settings, tend to value journal metrics such as Journal Impact Factor and h5-index, or author metrics such as $\mathrm{h}$ index or i10-index. Article metrics have traditionally been limited to statistics such as the number of journal citations, article downloads, or views on academic social media sites (e.g., ResearchGate).

The Kenya Medical Research Institute (KEMRI) conducted research to find out the 'Research Dissemination Strategies used by KEMRI scientists. Peer-reviewed journal articles were the most preferred method of communicating scientific outputs, while conferences and policy briefs were the others. They recommend that dissemination channels should go beyond simply making research widely available through traditional means and establish an institutional mechanism that would enable researchers to extract the main messages and key implications from their work (30).

\section{CONCLUSION}

This review presents an overview of the literature on importance of KT to researcher as well the obstacles it faces in African. The knowledge translation platforms in the WHO African countries can be described as suboptimal at best and non-existent at the worst. However, despite the barriers limiting the translation of research into practice, our observation and others supported the fact that research evidence is worthwhile for the desired improvements in health outcomes. Currently, translation of research findings into some concrete outputs which can affect health of people is not in mandate of researchers and subsequently they are not prepared for this as well. Based on the obstacles we identified, the following interventions are necessary: (a) cooperation among policy makers at macro and organizational level and the research sector. (b) establishing networks for researchers and decision makers in choosing the research topic, priority setting and (c) building trust among researchers and policy makers.

We also concluded that public health researchers are largely still concentrating on journal articles and scientific meetings for disseminating research findings to policy makers. These are important methods of dissemination, yet they do not link well with the needs and communication approaches that resonate with practitioners and policy makers. The following approaches could result in more effective dissemination and uptake of scientific health findings by the policy makers; (a) design studies in a way that emphasizes dissemination early in the research process through involvement of stakeholders,(b) enhance expectations from funders of research for more consistent and intentional dissemination (31),(c) identify and emphasize related incentives for dissemination in other organizations with a stake in dissemination for example creative approaches among publishers (32), (d) Shift the academic culture and incentives to include a greater focus on linking scientists with research users by involvement in policy making and practice placements for faculty members).

\section{RECOMMENDATION}

We recommend the inclusion of knowledge transfer advisors in protocols who will advocate of the knowledge translation process. Their roles may vary depending on the phases in the research and knowledge translation cycle. They support researchers throughout the research process, starting from the preparation of grant applications and in their interactions with workplace stakeholders.

\section{DECLARATION OF CONFLICTING INTERESTS}

The Authors declare that there is no conflict of interest. The opinions and assertions contained herein are the private opinions of the authors and are not to be construed as reflecting the views of Heart to Heart International (HHI).

\section{ETHICS APPROVAL}

Not applicable.

\section{FUNDING}

This research received no specific grant from any funding agency in the public, commercial, or not-forprofit sectors.

\section{REFERENCES}

[1] Graham ID, Logan J, Harrison MB, Straus SE, Tetroe J, Caswell W, et al. Lost in knowledge translation: time for a map? Journal of continuing education in the health professions. 2006;26(1):13-24.

[2] Green A, Bennett S. Sound choices: enhancing capacity for evidence-informed health policy: Geneva, World Health Organization; 2007.

[3] Poot CC, Van Der Kleij RM, Brakema EA, Vermond D, Williams S, Cragg L, et al. From research to evidence-informed decision making: a systematic approach. Journal of Public Health. 2018;40(suppl_1): i3-i12.

[4] Grimshaw JM, Eccles MP, Lavis JN, Hill SJ, Squires JE. Knowledge translation of research findings. Implementation science. 2012;7(1):117.

[5] Langlois EV, Montekio VB, Young T, Song K, Alcalde-Rabanal J, Tran N. Enhancing evidence informed policymaking in complex health systems: lessons from multi-site collaborative approaches. Health research policy and systems. 2016;14(1):1-11.

[6] Barratt H, Shaw J, Simpson L, Bhatia S, Fulop N. Health services research: building capacity to meet the needs of the health care system. Journal of health services research \& policy. 2017;22(4):243-9.

[7] Tetroe J. Knowledge translation at the Canadian Institutes of Health Research: a primer. Focus Technical Brief. 2007; 18:1-8. 
[8] Tetroe JM, Graham ID, Foy R, Robinson N, Eccles MP, Wensing $M$, et al. Health research funding agencies' support and promotion of knowledge translation: an international study. The Milbank Quarterly. 2008;86(1):125-55.

[9] Shroff Z, Aulakh B, Gilson L, Agyepong IA, ElJardali F, Ghaffar A. Incorporating research evidence into decision-making processes: researcher and decision-maker perceptions from five low-and middle-income countries. Health Research Policy and Systems. 2015;13(1):1-14.

[10] Kothari A, Regan S, Gore D, Valaitis R, Garcia J, Manson $\mathrm{H}$, et al. Using an integrated knowledge translation approach to build a public health research agenda. Health Research Policy and Systems. 2014;12(1):1-8.

[11] Lester L, Haby MM, Chapman E, Kuchenmüller T. Evaluation of the performance and achievements of the WHO Evidence-informed Policy Network (EVIPNet) Europe. Health research policy and systems. 2020;18(1):1-19.

[12] Organization WH. Working together for health: The World health report 2006: policy briefs: World Health Organization; 2006.

[13] Kasonde J, editor Report to International Development Research Centre: Development of the Zambia Forum for Health Research2009: Zambia Forum for Health Research, Lusaka, ZM.

[14] Berman J, Mitambo C, Matanje-Mwagomba B, Khan S, Kachimanga C, Wroe E, et al. Building a knowledge translation platform in Malawi to support evidence-informed health policy. Health research policy and systems. 2015;13(1):1-5.

[15] Hu B, Guo H, Zhou P, Shi Z-L. Characteristics of SARS-CoV-2 and COVID-19. Nature Reviews Microbiology. 2020:1-14.

[16] Nutley S, editor Challenges and opportunities of studying research-based knowledge use. Conférence publique présentée dans le cadre des activités de l'Équipe de recherche en partenariat sur le transfert de connaissances Montréal; 2011.

[17] Dagenais C. Research use at the Ministry of Health in Burkina Faso: the decision-makers' perspective. Implementation Science Communications. 2021;2(1):1-7.

[18] Pawson R. Evidence-based policy: a realist perspective: sage; 2006.

[19] Lavis JN, Robertson D, Woodside JM, McLeod $\mathrm{CB}$, Abelson J. How can research organizations more effectively transfer research knowledge to decision makers? The Milbank Quarterly. 2003;81(2):221-48.
[20] Kariuki JN, Kaburi J, Musuva R, Njomo DW, Night D, Wandera C, et al. Research Dissemination Strategies Used by Kenya Medical Research Institute Scientists. EA Health Research Journal. 2019;3(1):70-8.

[21] Ward V, House A, Hamer S. Knowledge brokering: the missing link in the evidence to action chain? Evidence \& policy: a journal of research, debate and practice. 2009;5(3):26779.

[22] Lomas J. Essay: Using 'Linkage and Exchange'To Move Research into Policy at A Canadian Foundation: Encouraging partnerships between researchers and policymakers is the goal of a promising new Canadian initiative. Health affairs. 2000;19(3):236-40.

[23] Straus SE, Tetroe J, Graham I. Defining knowledge translation. Cmaj. 2009;181(3-4):165-8.

[24] Kim T, Sharma M, Teerawattananon Y, Oh C, Ong L, Hangoma $\mathrm{P}$, et al. Addressing Challenges in Health Technology Assessment Institutionalization for Furtherance of Universal Health Coverage Through SouthSouth Knowledge Exchange: Lessons from Bhutan, Kenya, Thailand, and Zambia. Value in Health Regional Issues. 2021; 24:187-92.

[25] White E, Baddeliyanage R, Shaikh F, Dimaras H. Meaningful patient engagement in research: lessons from retinoblastoma. Pediatrics. $2019 ; 143(6)$.

[26] Esquivel-Sada D, Nguyen MT. Diagnosis of rare diseases under focus impacts for Canadian patients. Journal of community genetics. 2018;9(1):37-50.

[27] Zida A, Lavis JN, Sewankambo NK, Kouyate B, Ouedraogo S. Evaluating the process and extent of institutionalization: a case study of a rapid response unit for health policy in Burkina Faso. International journal of health policy and management. 2018;7(1):15.

[28] Eckl VC, editor Barriers of knowledge transfer. Paper submitted at DRUID Summer Conference; 2012.

[29] Santesso N, Tugwell P. Knowledge translation in developing countries. Journal of Continuing Education in the Health Professions. 2006;26(1):87-96.

[30] Shelton RC, Lee M, Brotzman LE, Wolfenden L, Nathan N, Wainberg ML. What is dissemination and implementation science?: an introduction and opportunities to advance behavioral medicine and public health globally. International journal of behavioral medicine. 2020;27(1):3-20. 
[31] Brownson RC, Eyler AA, Harris JK, Moore JB, Tabak RG. Research full report: getting the word out: new approaches for disseminating public health science. Journal of public health management and practice. 2018;24(2):102.
[32] Owen N, Healy GN, Dempsey PC, Salmon J, Timperio A, Clark BK, et al. Sedentary behavior and public health: integrating the evidence and identifying potential solutions. Annual review of public health. 2020; 41:265-87. 